\title{
Building a framework for evaluation of Fetal Alcohol Spectrum Disorder prevention and support programs: A collaborative Canadian project
}

\author{
Deborah Rutman ${ }^{1,2}$, Nancy Poole ${ }^{2,3}$, Sharon Hume ${ }^{1}$, Carol Hubberstey ${ }^{1}$, and Marilyn Van Bibber ${ }^{1,2}$ \\ ${ }^{1}$ Principal, Nota Bene Consulting Group, Victoria, Canada \\ ${ }^{2}$ Canada FASD Research Network, Network Action Team on Prevention, Vancouver, Canada \\ ${ }^{3}$ British Columbia Centre of Excellence for Women’s Health, Vancouver, Canada
}

\begin{abstract}
Aims: This article discusses a Canadian project that is designed to identify promising evaluation methods and create common evaluation frameworks for FASD prevention programs serving pregnant women and mothers, and FASD supportive intervention programs serving youth and adults living with FASD. A social determinants of health perspective guided the project.

Design: The project has employed a mixed-methods approach including a literature search, documentary review, and an iterative set of consultations with program providers, program managers, government managers and funders, researchers, and evaluators in the context of their work across Canada and internationally.

Results: The project's processes led to the development of three visual "maps" comprised of concentric rings that depict theoretical foundations; activities and approaches; formative outcomes; and participant, community and systemic outcomes. The three visual frameworks depict evaluation of 1) FASD prevention programs; 2) FASD support programs; and 3) FASD programs in Aboriginal communities.

Conclusions: The development of visual maps to depict common evaluation frameworks promotes individual and collective action towards applying the frameworks on the part of community-based services and governments across Canada, on the service and systemic levels. Program providers, researchers, and system planners have indicated that the maps have wide-ranging applications.
\end{abstract}

Addressing the social determinants of health of women who use substances before, during and after pregnancy is critical if Fetal Alcohol Spectrum Disorder (FASD) is to be prevented (Institute of Health Economics, 2009; Network Action Team on FASD Prevention from a Women's Health Determinants Perspective; 2010).

While a growing number of programs in Canada are employing a social determinants of health framework, there has been very limited independent evaluation of these programs, or of programs designed to support young people and adults living with FASD. Indeed, only a handful of comprehensive perinatal support services have been the subject of published evaluations, though notable exceptions in Canada include the Sheway program in Vancouver (Poole, 2000); the Breaking the Cycle program in Toronto (Motz, Leslie, Pepler, Moore, \& Freeman, 2006); the First Steps and Enhanced Services for Women programs in Edmonton (Watkins \& Chovanec, 2006); the Women's and Children's Healing and Recovery Program in Yellowknife
(Four World Centre for Development Learning, 2003); and the New Choices program in Hamilton (Niccols \& Sword, 2005). Systematic evaluations of programs geared to providing support to individuals living with FASD or their families are even more difficult to find in the literature, although very recently a few have been published, including evaluations of the Step by Step program in Alberta (Denys, Rasmussen, \& Henneveld, 2011) and the Key Worker program in British Columbia (Rutman, Hubberstey, \& Hume, 2011).

In addition, both within and across community-based services, to date there have been few opportunities for program planners, managers, staff, and funders to come together to discuss the goals and anticipated outcomes of programs that serve women at risk of having an alcoholexposed pregnancy, or to discuss goals and outcomes of programs that support individuals with FASD. This has

Correspondence: Deborah Rutman, 1434 Vining St., Victoria BC, V8R 1P8. Telephone: 250-370-6087. Fax: 250-721-6228. E-mail: drutman@uvic.ca Financial support: Public Health Agency of Canada, FASD Strategic Grants Fund

Keywords: Fetal Alcohol Spectrum Disorder, evaluation, FASD prevention, social determinants of health, multi-sectoral involvement 
meant that in Canada, as well as internationally, we lack common evaluation methods and outcome indicators. This absence of a common, well-articulated evaluation framework has made it difficult for program staff, planners and funders to identify which aspects of programs contribute to positive client and community outcomes. Our lack of a common evaluation framework has also hampered efforts to conduct multi-site studies to identify whether or how programs contribute to FASD prevention, improve the health of women and/or those living with FASD, and help prevent or reduce secondary and tertiary effects of FASD.

To address this knowledge gap, the Toward an Evaluation Framework for Community-based FASD Prevention Programs project was conceived with the intent of bringing researchers, evaluators, program managers and staff, and funders together to identify promising evaluation methods and to create common evaluation frameworks and tools for FASD prevention programs serving pregnant women and mothers, and for FASD supportive intervention programs ${ }^{1}$ serving youth and adults living with FASD. As well, in view of the reality that community-based programs face mounting pressure to undertake evaluation-often with minimal or no additional resources - the project has aimed to create evaluation-related resources that can be used easily by community-based programs. In this way, the project aims to enhance community-based evaluation capacity and also take into consideration the diversity of the programs' cultural, geographic, economic, and service delivery contexts.

\section{Method}

The project's five-person team has expertise in FASD prevention and research, in the evaluation of FASD-related programs, and in working with and for Aboriginal communities to address issues related to the social determinants of health and community-based FASD prevention. The project also has been guided by a national Advisory Committee, comprised of 13 people with expertise in prevention, FASD and evaluation.

Our philosophical starting point is to view program evaluation as a resource to inform evidenced-based decision-making, and as a means to increase learning about the following: how a particular model works with a given population; how program improvements can be made, and how to provide feedback to enhance program effectiveness; how new outcomes and outcome measures may be identified, as our understanding of the needs of a given population increases; and what difference a program is

\footnotetext{
${ }^{1}$ In this project, we use the terms "FASD-supportive intervention programs" and "FASD support programs" interchangeably, and we have defined these as programs that aim to support and/or assist people living with FASD and their families and support networks to improve knowledge, skills and community connections, so as to better address issues associated with day-to-day living. These programs differ from FASD intervention programs in that they are not primarily focused on addressing or ameliorating the primary effects of FASD (e.g., improving aspects of cognitive functioning known to be particularly affected by prenatal exposure to alcohol).
}

making for participants, providers, communities and service systems. In keeping with others engaged in community-based and developmental evaluation, we also believe that program evaluation is not about judging success versus failure; program inspection; or measuring programs against fixed goals (Hutchinson \& van der Woerd, 2010; Patton, 2011).

The project's multi-method process has involved a number of integrated and iterative data-collection activities. As an initial step, we contacted program providers, researchers, and evaluators across Canada and internationally in order to identify and gather published and unpublished evaluations of FASD prevention and FASD support programs, including evaluations of pregnancy outreach programs; parent mentoring programs (e.g., Parent-Child Assistance Program); supportive intervention programs for youth or adults living with FASD; programs focusing on addressing social determinants of health for pregnant and parenting women; and FASD prevention or support programs within Aboriginal communities.

We then reviewed these published and unpublished evaluation reports and related materials, guided by the following questions:

- How were FASD prevention and intervention programs serving women and their families being evaluated? What methodologies and methods of data collection were employed?

- What were identified as key program activities and approaches, and what were the theoretical and/or philosophical underpinnings of the programs?

- What were identified as key participant, program and community outcomes, including both formative and summative, and short-term, intermediate and longterm outcomes?

- What were programs' indicators of these outcomes; that is, what were markers of success as well as key program outputs?

- What data collection tools were used in the evaluations?

- What, if anything, did evaluators identify as being promising approaches to evaluation?

In our efforts to organize the rich and detailed information that resulted from our consultations and documentary/ literature review, we began to appreciate that visual as well as tabular displays of the data would be useful. Indeed, the team recognized that there was value in creating a visual means of mapping the "big picture"-including programs' theoretical foundations, program activities and approaches, program/formative outcomes, participant outcomes, community outcomes, and systemic outcomes. This led us to draft three separate visual "maps," depicting evaluation of 1) FASD prevention programs; 2) FASD support programs; and 3) FASD programs in Aboriginal communities. 
The next key facet of our project process was to obtain feedback on our emerging evaluation frameworks ${ }^{2}$. Accordingly, in the fall of 2011 and in spring/summer of 2012, the team facilitated day-long consultations with program providers and researchers in six regions of Canada. During these meetings, we received feedback on the draft evaluation maps, and then, in an iterative manner, made revisions to the maps following each regional session. In addition, in 2012 the emerging evaluation frameworks were presented at several national FASDrelated conferences, which have become additional opportunities to invite feedback on the visual design of the maps and on all related elements. As of this writing, approximately 250 people from all regions in Canada have participated in consultations and presentations related to the project.

Two other components are key to the project's process and second year (2012/2013). First, the project team has been creating a website as a resource for FASD program evaluation (www.fasdevaluation.ca); posted will be the operational definitions, outcomes, indicators, possible measurement tools, and other relevant content specially created for each of the three maps. Second, the project is working with several FASD prevention, support and Aboriginal programs across Canada and is offering mentoring and evaluation-related support to staff and managers and, in some cases, funders and researchers connected to these programs. The mentoring is focused on helping programs address whatever evaluation-related questions or issues are of most concern. In most cases, however, the evaluation mentoring will provide community-based programs with opportunities to develop and/or refine their own program-specific evaluation frameworks and identify outcomes, indicators and data collection processes and tools that are most relevant to them. It is anticipated that the project's Evaluation Maps will be used to frame evaluation activities.

\section{Results}

The striking feature of all three maps' design is their circular structure; this immediately distinguishes them from more typical evaluation frameworks or logic models that tend to use a linear matrix or grid-like layout. Indeed, the circular design is congruent with, and has been informed by, Indigenous wheel-based frameworks of well-being that emphasize the inter-connectedness of all aspects of existence, phases of the lifespan and future generations, as well as the importance of wholistic approaches to healing and understanding (Kryzanowski \& McIntyre, 2011).

At the center of the evaluation map for FASD prevention programs, in its innermost ring, is the "Pregnant woman/mother and child" (see Figure 1). This reflects our understanding that FASD prevention programs need to focus on both the woman and her child (or fetus) - both are the "clients." Indeed, one pioneering Canadian FASD prevention program, Breaking the Cycle in Toronto, identifies the woman, the child, and the connection between mother and child as the program's "client" (Motz et al, 2005). Placing the pregnant woman/mother and child at the center of the map also reflects programs' participantcentered approach to care, an element that is reiterated in the ring of our maps that represents a program's philosophical/theoretical components.

Surrounding the "Pregnant woman/mother and child," in the second-innermost ring, is "Family and Community Support." Placing family and community support in the near-center of the framework is essential, as this reflects the central importance of family, including the women's partner, community, and culture, in relation to her wellbeing and healing. As well, the chromatically linked tones of the two innermost rings in the evaluation map are intended to emphasize the inter-connections between the woman and her child, and her family and community support.

The next ring of our map focuses on the elements of a program's philosophy and/or theoretical framework (see Figure 2). By identifying key elements of FASD prevention and support programs' philosophy and highlighting these elements as core to the evaluation framework, the maps are again different from more conventional evaluation frameworks or logic models. Indeed, our review of existing evaluation literature related to FASD programming confirmed the fact that, unfortunately, programs' philosophical underpinnings often are not articulated, despite their importance in guiding program activities and approaches.

\section{Figure 1}

Evaluation map for FASD Prevention Programs: Focusing on the inner two rings

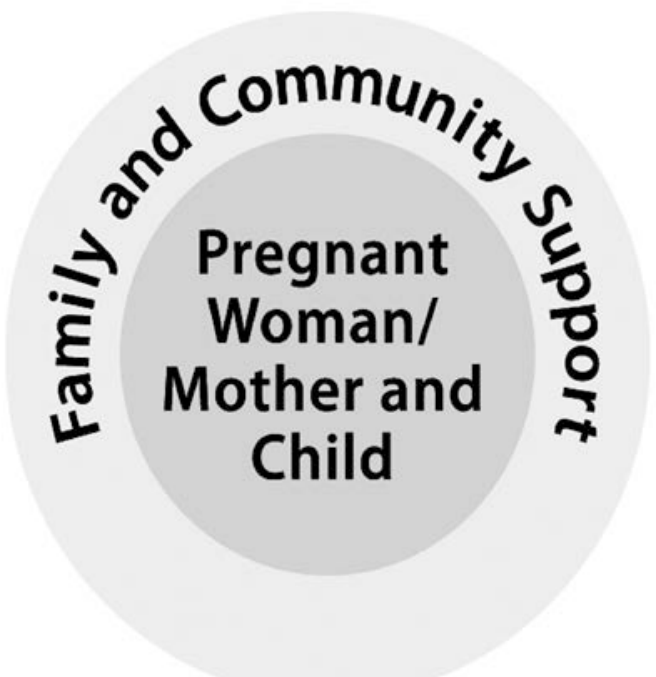

${ }^{2}$ Please note that in this discussion and in the Findings section we are using the terms Evaluation Map and Evaluation Framework interchangeably. 
Figure 2

Evaluation map of FASD Prevention Programs: Focusing on the Program Philosophy/Theoretical Framework ring

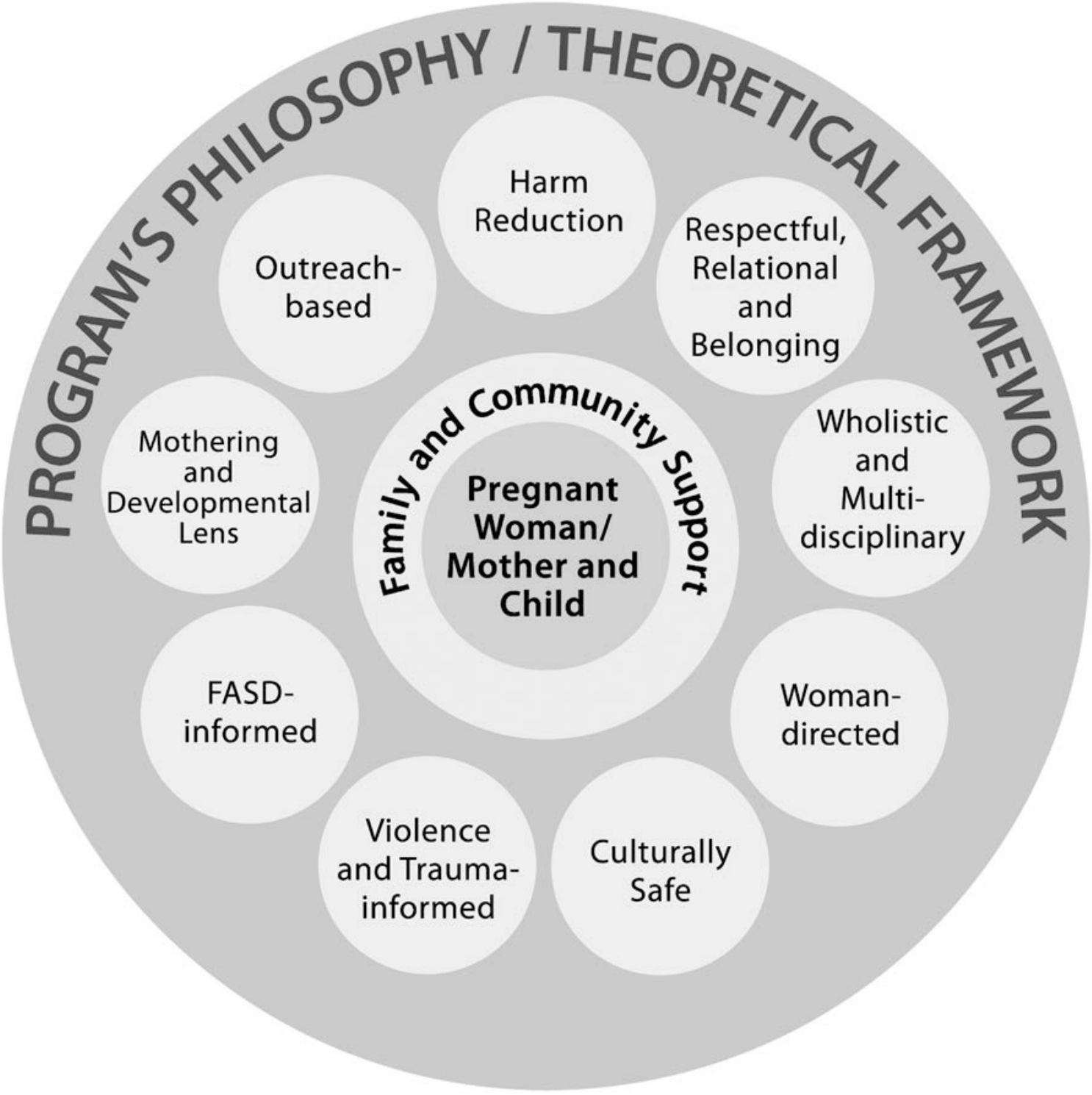

In this evaluation map for FASD prevention programs, we have identified nine elements that may be part of a program's philosophy/theoretical framework: FASDinformed lens; Mothering \& developmental lens; Culturally safe; Wholistic and multi-disciplinary; Respectful, relational, belonging; Participant/family-directed; Violence and trauma-informed; Harm reduction; and Outreachbased. It is important to emphasize that we are not suggesting that all FASD prevention (or support) programs need to be based on all nine of these elements; rather, our map depicts an array of philosophical/theoretical elements that Canadian and international FASD prevention programs have articulated as being important to the delivery of services and support to pregnant women and their children and/or adults living with FASD.

Our project's website will provide definitions and potential indicators of each element, including data collection tools that may enable evaluators and program staff to assess whether a program is delivering services in keeping with a given philosophical/theoretical element, such as being "violence and trauma-informed." While it is beyond the scope of this article to provide definitions and indicators of all elements, for illustrative purposes we share in Table 1 a set of potential indicators of an "FASD-informed lens," based on our analysis of the relevant evaluation-related and promising-practices literature. 
Table 1

\section{Potential Indicators of an FASD-informed approach}

Using an FASD-informed approach, programs and service providers:

- have training in FASD

- use person-first language ("child with FASD,” not "FASDchild")

- employ a relational and strengths-based approach

- gear practice to developmental age

- make accommodations to communication, program format, and physical environment

- use an individualized care plan and one-to-one support

- have ongoing FASD-focused supervision

- are resourced to enable smaller caseloads.
Moving out from "Philosophical/Theoretical Framework," the next ring in our evaluation map is "Activities/ Approaches" (see Figure 3). Here, we identify an array of activities that FASD prevention programs undertake, assembled based on our review of evaluation reports, program documents and our regional consultations. Again, we emphasize that our map does not prescribe that all FASD prevention programs should carry out all these activities. However, reflecting on these activities enables program staff, planners, funders and researchers/evaluators to recognize the number and types of activities that can comprise effective FASD prevention programs. As well, by specifying these activities, we also make visible what in some cases are invisible-and unfunded-program activities, such as transportation, client accompaniment to meetings, and provision of food.

\section{Figure 3}

\section{Evaluation map of FASD Prevention Programs: Focusing on the Program Activities/Approaches ring}

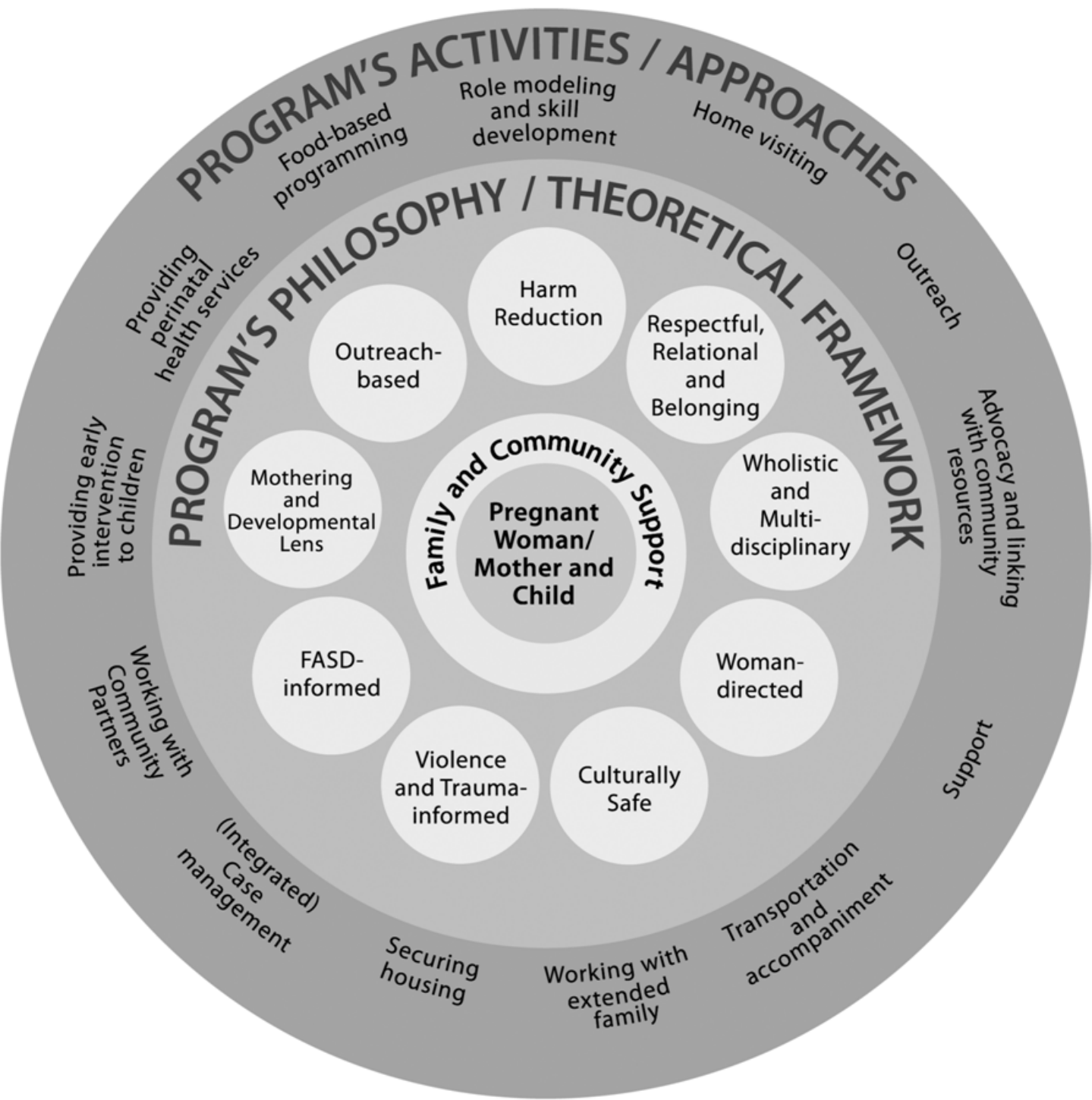


As shown in Figure 4, the next ring of the map, "Program Outcomes," identifies four key categories of formative outcomes that pertain to FASD prevention programs. On our project's website, we expand on this by identifying specific program outcomes within each of the four headings, including:

- participants have positive experiences in the program (e.g., program feels welcoming, safe, respectful)

- participants take part in decision making

- the program is accessible

- the program is flexible

- staff have adequate, relevant training, support and supervision
- staff employ informed approaches (i.e., violence and trauma-informed, FASD-informed, culturally safe)

- the team works collaboratively, and

- there is funding adequacy.

The project's website (www.fasdevaluation.ca) also will provide indicators and tools to guide data collection related to these program outcomes. This ring of our evaluation map is thus quite important, as it threads the elements of the "Philosophical/Theoretical Framework" ring with outcomes related to participants' and service providers' experience of the program, as well as with systemic outcomes.

\section{Figure 4}

Evaluation map of FASD Prevention Programs: Focusing on the Program Outcomes ring

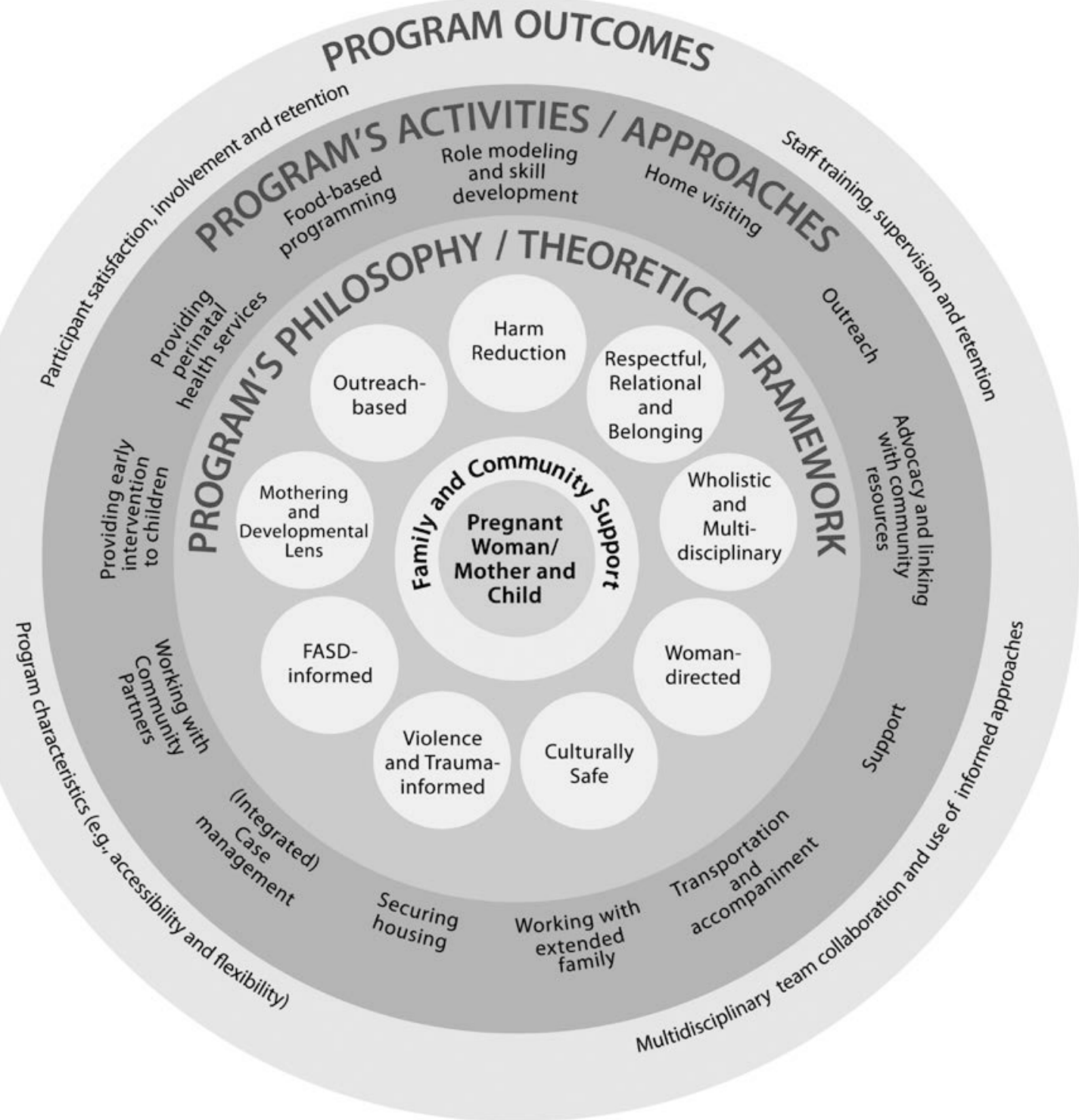


Lastly, the two outermost rings of the evaluation map identify summative outcomes for participants (i.e., outcomes that relate to the evaluation question "What difference did the program make to participants?"), and then outcomes at a community and a systemic level (see Figure 5). As emphasized above, our aim is not to suggest that each and every FASD prevention program need strive to achieve all the participant outcomes named in the circles of this ring; rather, our map aims to provide information regarding the array of outcomes that FASD prevention programs collectively have identified as being desired and/or achieved as a result of their program. An additional feature of the design of the "Participant Outcomes" ring in our maps is the suggestive clustering of outcomes by quadrants recognized within Indigenous frameworks (e.g., spiritual and mental), which again reflects our intent to emphasize the importance of conceptualizing program delivery, and participant needs and outcomes, from a wholistic perspective.

\section{Figure 5}

\section{Evaluation map of FASD Prevention Programs}

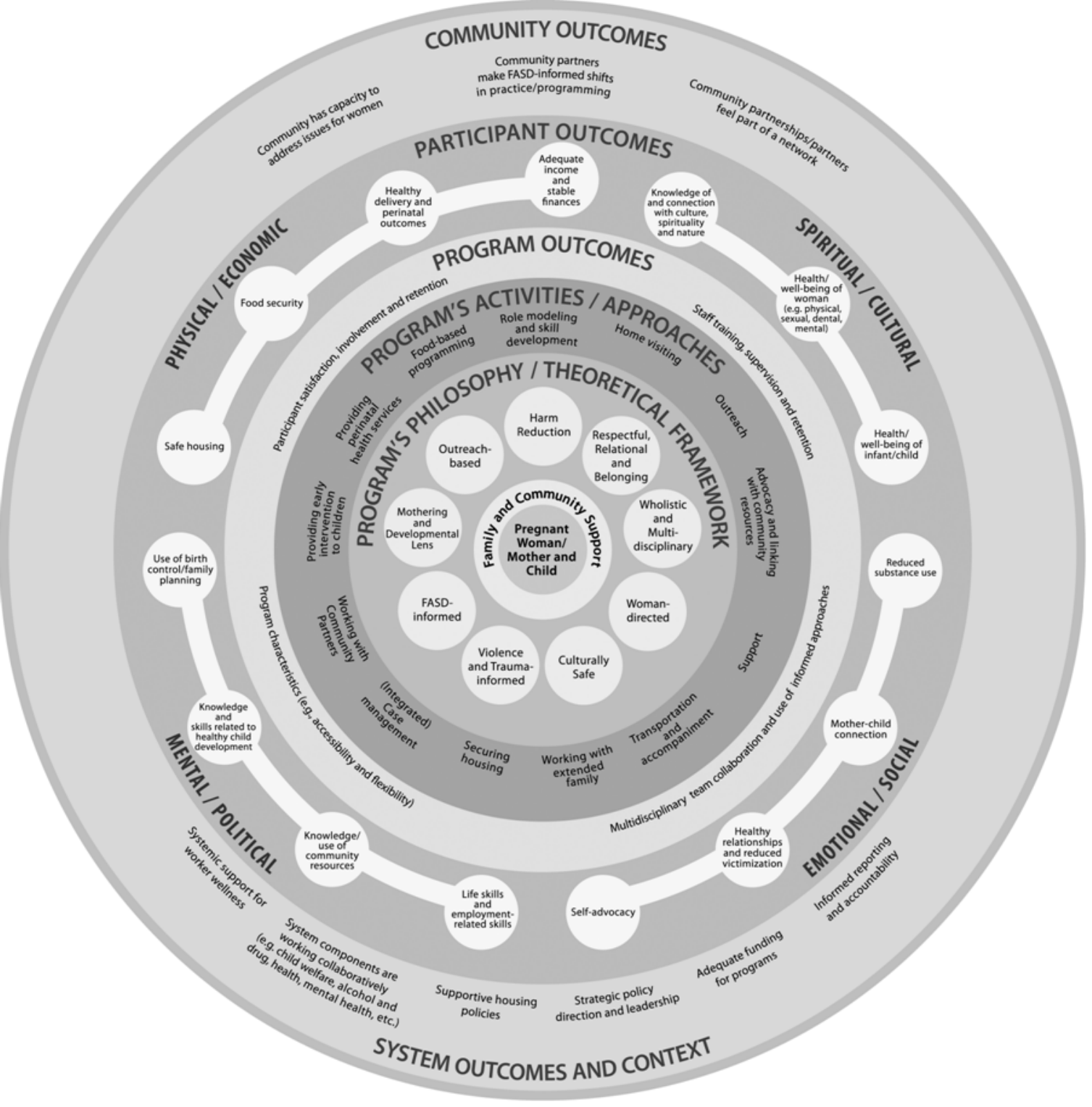


While this article has focused on describing the elements comprising the Evaluation Map for FASD Prevention Programs, the project has also produced an Evaluation Map for FASD Support Programs and a Map for Evaluating FASD Programs in Aboriginal Communities. Although the overall design and majority of elements are shared between the three maps, there are also some important differences. For example, the evaluation map pertaining to FASD programs in Aboriginal communities has "Culture" in the innermost ring, which is then encircled by "Family, Child, Youth, Adult, and Elder" to denote the centrality of culture and extended family in the provision of support leading to wellness and healing. The map for FASD support programs has "Youth/Adult with FASD" in the innermost ring, surrounded by "Family and Community Support," to emphasized the importance of an individualized approach to working with people living with FASD.

\section{Applications and Implications}

The circular evaluation maps developed through this project represent a departure from the more traditional linear or matrix style of evaluation frameworks. It is a departure that has been received enthusiastically throughout the consultation process. The wholistic approaches, as reflected by the circles and the four aspects of wellnessspiritual, mental, physical and emotional-espoused by Indigenous peoples, have been an important foundation to the work, respecting and addressing the scope of influences on women's drinking and the range of needs of those with FASD.

Moreover, interest in contributing to the maps through the consultation sessions has reinforced how evaluation can be a co-learning process. The process of gathering input from diverse groups of front-line workers, managers, funders, researchers and evaluators working with a range of FASDrelated programs from across Canada has highlighted both the strength of a social determinants of health approach in program development and delivery, and the commonalities and important differences across FASD prevention and support programs-including FASD programs in Aboriginal communities-regarding philosophy, activities, and outcomes.

The evaluation maps have generated excitement about engaging in evaluation activities - a shift for some who previously had been wary or intimidated by the prospect of program evaluation. The circular nature of the maps, which captures and succinctly links the different components of evaluation, clearly resonates. The maps are being integrated with programs' existing evaluation activities, even without the more detailed information related to indicators and data collection tools that is to be posted on the project's website.

Program providers have reported that the evaluation maps have inspired them to reflect on their program philosophy and how they might translate these principles into practice. The maps have helped them become more articulate about why certain activities are provided, and have revealed areas where there may be gaps. System planners have expressed interest in examining how their work can respect the wide range of outcomes, rather than insisting on a narrow scope of system-generated outcomes that may not accurately capture all that the programs achieve. Researchers see the benefit for potential cross-site research strategies employing similar frameworks to generate evidence about what works and what makes a difference for those affected by FASD. Together, the interest and participation of diverse groups in refining the frameworks affirm the multisectoral approach to building a body of knowledge about what contributes to positive outcomes for clients and communities.

\section{References}

British Columbia Reproductive Care Program. (1999). Principles of perinatal care for substance-using women and their newborns. Vancouver, BC: Author.

Denys, K., Rasmussen, C. \& Henneveld, D. (2011). The effectiveness of a community-based intervention for parents with FASD. Community Mental Health Journal, 47, 209-219.

Four Worlds Centre for Development Learning. (2003). Making the path by walking it: A comprehensive evaluation of the Women and Children's Healing and Recovery Program (WCHRP) Pilot. Yellowknife, NT: WCHRP.

Hutchinson, K. \& van der Woerd, K. (2010). Basics of community-based evaluation. Retrieved from: http://ebookbrowse.com/caiwebinar-basics-ofcommunity-based-evaluation-nov-16-2010-pdfd36769698

Institute of Health Economics. (2009). Consensus statement on Fetal Alcohol Spectrum Disorder (FASD)-Across the lifespan. Edmonton, $\mathrm{AB}$ : Institute of Health Economics and Government of Alberta.

Kryzanowski, J. \& McIntyre, L. (2011). A holistic model for the selection of environmental assessment indicators to assess the impact of industrialization on Indigenous health, Canadian Journal of Public Health, 102, 112-117.

Motz, M., Leslie, M., Pepler, D., Moore, T., \& Freeman, P. (2006). Breaking the Cycle: Measures of progress 1995-2005, Special Supplement Journal of FAS International, 4:e22. Toronto, ON: Hospital for Sick Children.

Network Action Team on FASD Prevention from a Women's Health Determinants Perspective. (2010). 10 fundamental components of FASD prevention from a women's health determinants perspective. Vancouver, BC: Canada Northwest FASD Research Network and BC Centre of Excellence for Women's Health. Retrieved from http://www.canfasd.ca/files/PDF/ ConsensusStatement.pdf.

Niccols, A. \& Sword, W. (2005). "New Choices" for substance-using mothers and their children: Preliminary evaluation. Journal of Substance Use, 10, 239-251.

Patton, M. (2011). Developmental evaluation: Applying complexity concepts to enhance innovation and use. New York, NY, United States: The Guilford Press. 
Rutman, D., Hubberstey, C. \& Hume, S. (2011). British Columbia’s Key Worker and Parent Support Program: Evaluation highlights and implications for practice and policy. In E. Riley, S. Clarren, J. Weinberg \& E. Jonsson (Eds.) Fetal Alcohol Spectrum Disorder: Management and Policy Perspectives of FASD. Weinheim, Germany: Wiley-VGH Verlag \& Co.

Watkins, M., \& Chovanec, D. (2006). Women working toward their goals through AADAC Enhanced Services for Women. Edmonton, AB: AADAC 